\title{
On the solution of an integral equation of second type by the Monte-Carlo method
}

\author{
by NGUYeN QuY Hy (Warszawa)
}

\begin{abstract}
In this work, the Markov processes and the corresponding random variable are constructed. The sum of the expected values of these random variable is the solution of an integral equation of the form: $u(x)-\int_{Q} K(x, y) u(y) \mu(d y)=g(x)$ in the space of $\Sigma$-measurable and bounded in $\Omega(\bmod \mu)$ functions. Also, for the calculation of linear functionals (Hilbert space inner products) determined by the solutions of the mentioned integral equations.
\end{abstract}

1. Introduction. In this paper, the solution of the following integral equation in the space $L_{\infty}(\Omega)\left(^{1}\right)$ is obtained with use of the Monte-Carlo method:

$$
u(x)-\int_{\Omega} K(x, y) u(y) \mu(d y)=g(x) \quad(x \in \Omega)
$$

where $(\Omega, \Sigma, \mu)$ is a measure space, $\mu(\Omega)<+\infty$.

Suppose there exists a set $\Omega_{0} \in \Sigma$ such that $\Omega_{0} \subset \Omega, \mu\left(\Omega_{0}\right)>0$ and it fulfils the following conditions:

(A) For all $f \in L_{\infty}\left(\Omega \backslash \Omega_{0}\right)\left({ }^{1}\right)$, the series $\sum_{n=0}^{\infty} T_{+}^{n} f$ converges in $L_{\infty}\left(\Omega \backslash \Omega_{0}\right)$, where the integral operator $T_{+}$is defined by the formula:

$$
\left[T_{+} f\right](x)=\int_{\Omega \backslash \Omega_{0}}|K(x, y)| f(y) \mu(d y) \quad\left(x \in \Omega \backslash \Omega_{0}\right),
$$

(B) $K(x, y) \geqslant 0$ for: $x \epsilon \Omega \backslash \Omega_{0}(\bmod \mu), y \epsilon \Omega_{0}(\bmod \mu)$,

(C) $\quad K(x, y)=$ for $: x \in \Omega_{0}(\bmod \mu), y \in \Omega(\bmod \mu)$.

Here $\Omega$ is any abstract set. In particular, when $\Omega$ is a discrete set, equation (1.1) reduces to a system of linear algebraic equations or a system of difference equation.

(1) The notation $L_{\infty}(A)$ denotes the space of $\Sigma$-measurable and hounded on $A(\bmod \mu)$ functions, where $A \in \Sigma$. 
The Monte-Carlo method is also used in this paper to obtain an estimation of the value of the functional:

$$
(u, \varphi) \equiv \int_{\Omega} u(x) \varphi(x) \mu(d x)
$$

where $u(x)$ is the solution of equation (1.1) and $\varphi \epsilon L_{1}(\Omega)\left({ }^{2}\right)$.

As is well known, the study of the functional of the form (1.2) is connected with the solution of the boundary problems by the potential method. Moreover, the estimation of the functional (1.2), in some special cases, has important consequences in the theory of the transfer equation of nuclear physics ([9], [10], [11]).

In Refs [8], [9], [10] various probability models have been considered in some special cases for the solution of the problems (1.1), (1.2). In [13] a particular case of the problems (1.1), (1.2) has been studied without conditions (B), (C) and a number of probability models has been given as the generalization of those mentioned in the works [7], [4] [12]( $\left.{ }^{3}\right)$.

The mentioned models are based essentially on the constiuction of a certain Markov chain and corresponding random variable, whose expected value is the solution of the problem.

In this paper the results of [2], [3] are generalized so as to apply to a construction of two Markov processes and the corresponding random variables in such a way that the sum of the expected values of these random variables will be the solution of the problem (1.1), (1.2).

2. The probability model for the solution of the integral equation (1.1). Suppose that there exists a measurable function $p(x, y)$, bounded on $\Omega \times \Omega$ and satisfying the following conditions:

$$
\begin{gathered}
\alpha \equiv \underset{\mu}{\operatorname{vrai}} \sup _{x \in \Omega \backslash \Omega_{0}}\left\{\int_{\Omega} p(x, y) K(x, y) \mu(d y)\right\}<1 ; \\
p(x, y) K(x, y) \geqslant 0 \quad \text { if } x \epsilon \Omega(\bmod \mu), y \in \Omega(\bmod \mu) ; \\
p(x, y) \neq 0 \quad \text { if }(x, y) \in \Omega \times \Omega(\bmod \mu \times \mu) .
\end{gathered}
$$

Then the following lemma is evident:

LEMMA (2.1). Suppose that the functions $p(x, y), K(x, y)$ satisfy conditions $(\mathrm{B}),\left(\mathrm{P}_{1}\right),\left(\mathrm{P}_{2}\right)$ and $g \in L_{\infty}(\Omega)$. Then there exists a set $A^{*}{ }_{\epsilon} \Sigma$ such that $\mu\left(A^{*}\right)=0$ and the following conditions are satisfied:

(2) $L_{1}(A)$ is the space of $\mu$-integrable functions on $A$, where $A \in \Sigma$.

(3) As it is known (see [6]), if we replace $L_{\infty}$ by $L_{p}(p>1)$ and $L_{1}$ by $L_{q}(1 / p+$ $+1 / q=1$ ), then we can use these models for the solution of the problems (1.2), (1.1) with $\Omega \subset R^{n}, \mu=\mathscr{L}$ ( $\mathscr{S}$ is the Lebesgue measure on $R^{n}$ ). 
(B*) $\quad K(x, y) \geqslant 0$ for $x \in \Omega_{A}^{*} \backslash \Omega_{0}, y \in \Omega_{0}(\bmod \mu)$, where

$\left(P_{1}^{*}\right)$

$$
\Omega_{A}^{*}=\Omega \backslash A^{*} ;
$$

$$
a^{*} \equiv \sup _{x \in \Omega_{A}^{*} \backslash \Omega_{0}}\left\{\int_{\Omega} p(x, y) K(x, y) \mu(d y)\right\}<1 ;
$$

$\left(P_{2}^{*}\right)$

$$
p(x, y) K(x, y) \geqslant 0 \quad \text { for } x \in \Omega_{A}^{*}, y \in \Omega(\bmod \mu) .
$$

Moreover,

$$
G \equiv \sup _{x \in \Omega_{A}^{*} \backslash \Omega_{0}}\{|g(x)|\}<+\infty .
$$

LEMMA (2.2) Let the assumptions of Lemma (2.1) be satisfied; suppose that $\delta, \Delta\left(^{4}\right)$ are certain positive constants and:

$$
\begin{gathered}
g_{1}(x)=-\left[|g(x)|+\chi_{\Omega_{0}}(x)\left(\frac{2 G M}{1-a^{*}}+\Delta\right)\right] \quad(x \in \Omega), \\
g_{2}(x)=g(x)-g_{1}(x) \quad(x \in \Omega), \\
h_{i}(x)=\frac{1}{\delta}\left[1-\int_{\delta} p(x, y) K(x, y) \mu(d y)-\frac{g_{i}(x)}{\mu\left(\Omega_{0}\right)} \int_{\Omega_{0}} \frac{p(x, y) \mu(d y)}{g_{i}(y)}\right] \\
\left(i=1,2 ; x \in \Omega_{A}^{*} \backslash \Omega_{0}\right),
\end{gathered}
$$

where $M$ is the constant defined by the formula:

$$
M \equiv \sup _{(x, y) \in\left(\Omega_{A}^{*} \backslash \Omega_{0}\right) \times \Omega_{0}}\{|p(x, y)|\}<+\infty
$$

Then we have $g_{1}, g_{2} \in L_{\infty}(\Omega)$ and

$$
\begin{gathered}
\frac{g_{i}(x)}{g_{i}(y)} \geqslant 0 \quad(i=1,2) \text { for }(x, y) \in\left(\Omega_{A}^{*} \backslash \Omega_{0}\right) \times \Omega_{0}, \\
h_{i}(x) \geqslant 0 \quad(i=1,2) \text { for } x \in \Omega_{A}^{*} \backslash \Omega_{0},
\end{gathered}
$$

Moreover, $h_{i}(x)(i=1,2)$ are $\Sigma$-measurable functions, bounded on $\Omega_{A}^{*} \backslash \Omega_{0}$.

Proof. From (2.3), (2.4) we have immediately formula (2.7) and $g_{1}, g_{2} \epsilon L_{\infty}(\Omega)$.

From (2.2), (2.3) we have

$$
g_{1}(y)<-\left[|g(y)|+\frac{2|g(x)| M}{1-a^{*}}\right] \quad\left(x \in \Omega_{A}^{*} \backslash \Omega_{0}, y \in \Omega_{0}\right) .
$$

${ }^{(4)}$ Under condition $\left(\mathrm{P}_{3}\right)$, we may replace the assumption $\Delta>0$ by the following ones:

$$
\begin{aligned}
& \Delta>0 \text { if } g(x) \not \equiv 0 \text { on } \Omega \backslash \Omega_{0}(\bmod \mu), \\
& \Delta>0 \text { if } g(x) \equiv 0 \text { on } \Omega \backslash \Omega_{0}(\bmod \mu) .
\end{aligned}
$$

5 - Annales Polonicl Mathematicl XXX.2 
Hence by (2.6) we get

$$
\frac{g_{1}(x) p(x, y)}{g_{1}(y)} \leqslant \frac{-g(x) M}{g_{1}(y)}<1-\alpha^{*} \quad\left(x \in \Omega_{A}^{*} \backslash \Omega_{0}, y \in \Omega_{0}\right) .
$$

Using condition $\left(\mathrm{P}_{1}^{*}\right)$ and $(2.10),(2.5)$ we have

$$
h_{1}(x)>0 \quad\left(\text { for } x \in \Omega_{A}^{*} \backslash \Omega_{0}\right) \text {. }
$$

Moreover, from (2.4), (2.9), (2.6) we obtain

$$
\frac{g_{2}(x) p(x, y)}{g_{2}(y)} \leqslant \frac{2|g(x)| M}{g_{2}(y)}<1-a^{*} \quad\left(x \in \Omega_{A}^{*} \backslash \Omega_{0}, y \in \Omega_{0}\right) .
$$

Consequently, we have in analogy with (2.10)

$$
h_{2}(x)>0 \quad\left(\text { for } x \in \Omega_{A}^{*} \backslash \Omega_{0}\right) \text {. }
$$

In addition, from (2.10), (2.11) and condition $\left(\mathrm{P}_{1}^{*}\right)$ we deduce that $h_{i}(x)(i=1,2)$ are $\Sigma$-measurable and bounded functions on $\Omega_{A}^{*} \backslash \Omega_{0}$. This completes the proof.

If we extend and complete the given measure $\mu$ defined on the $\sigma$-field $\Sigma$, then we obtain the complete measure $\bar{\mu}$ defined on the $\sigma$-field $\bar{\Sigma}$. In addition, $\bar{\mu}(\Omega)<+\infty$ for $\mu(\Omega)<+\infty$. Then we have

LEMMA (2.3). Suppose that

$$
\tilde{\Omega}=\Omega \cup \Omega^{*} ; \quad \tilde{\Sigma}=\bar{\Sigma} \cup \Sigma^{*} ; \quad \tilde{\mu}(\tilde{A})=\bar{\mu}(\tilde{A} \cap \Omega)+\delta \cdot \delta_{\tilde{A}} \quad(\nabla \tilde{A} \epsilon \tilde{\Sigma}),
$$

where $\Omega^{*}$ is a set with $\Omega^{*} \neq \emptyset, \Omega^{*} \cap \Omega=\emptyset$ and $\delta$ is the constant defined in Lemma (2.2);

$$
\begin{gathered}
\Sigma^{*}=\left\{\tilde{A}: \tilde{A}=\bar{A} \cup \Omega^{*}, \bar{A} \in \bar{\Sigma}\right\} ; \\
\delta_{\tilde{A}}= \begin{cases}1 & \text { if } \bar{A} \cap \Omega^{*} \neq \emptyset, \\
0 & \text { if } \bar{A} \cap \Omega^{*}=\emptyset .\end{cases}
\end{gathered}
$$

Then $(\tilde{\Omega}, \tilde{\Sigma}, \tilde{\mu})$ is a space with complete measure. Moreover,

$$
\begin{aligned}
& \tilde{\mu}\left(\Omega^{*}\right)=\delta, \quad \tilde{\mu}(\tilde{\Omega})<+\infty, \\
& \tilde{\mu}(A)=\mu(A) \quad(\text { for } A \in \Sigma) .
\end{aligned}
$$

Proof. From (2.12), (2.13), it is easy to see that $\tilde{\Sigma}$ is a $\sigma$-filed of subsets of $\tilde{\Omega}$. We shall show that for any sequence of setş $\tilde{A}_{k} \in \tilde{\Sigma}(k=1,2, \ldots)$, we have

$$
\tilde{\mu}\left(\bigcup_{k=1}^{\infty} \tilde{A}_{k}\right)=\sum_{k=1}^{\infty} \tilde{\mu}\left(\tilde{A}_{k}\right) \quad\left(\text { where } \tilde{A}_{i} \cap \tilde{A}_{k}=\emptyset ; i, k=1,2, \ldots\right) .
$$

In the case: $\bar{A}_{k} \in \bar{\Sigma}(k=1,2, \ldots)$, as is seen from (2.12), formula (2.17) is obvious. 
Suppose there exists an element of the sequence $\left\{\tilde{A}_{k}\right\}$. This element belongs to the class $\Sigma^{*}$.

Denote it by $\tilde{A}_{1}$. Then it is clear that (see formula (2.13))

$$
\tilde{A}_{1}=\bar{A}_{1} \cup \Omega^{*} \quad\left(\text { where } \bar{A}_{1} \in \bar{\Sigma}\right) \text {. }
$$

In this case, all the sets of the sequence $\left\{\tilde{A}_{k}\right\}$, except $\widetilde{A}_{1}$, belong to $\bar{\Sigma}: \tilde{A}_{2}, \tilde{A}_{3}, \ldots \in \bar{\Sigma}$.

Therefore from (2.12), (2.18) we deduce

$$
\begin{aligned}
\tilde{\mu}\left(\bigcup_{k=1}^{\infty} \tilde{A}_{k}\right) & =\tilde{\mu}\left\{\left[\bar{A}_{1} \cup\left(\bigcup_{k=2}^{\infty} \tilde{A_{k}}\right)\right] \cup \Omega^{*}\right\}=\bar{\mu}\left(\bar{A}_{1}\right)+\sum_{k=2}^{\infty} \tilde{\mu}\left(\tilde{A}_{k}\right)+\delta \\
& =\tilde{\mu}\left(\tilde{A_{1}}\right)+\sum_{k=2}^{\infty} \tilde{\mu}\left(\tilde{A}_{k}\right)=\sum_{k=1}^{\infty} \tilde{\mu}\left(\tilde{A}_{k}\right)
\end{aligned}
$$

i. e. we obtain (2.17).

Moreover, we see from (2.12) that $\tilde{\mu}(\emptyset)=0 ; \tilde{\mu}(\tilde{A}) \geqslant 0$ (for all $\tilde{A} \epsilon \bar{\Sigma}$ ). Therefore $\tilde{\mu}$ is a measure defined on $\tilde{\Sigma}$. Since $\bar{\mu}$ is a complete and finite measure on the $\sigma$-field $\bar{\Sigma}$, it is not difficult to deduce (see formula (2.12)) that $\tilde{\mu}$ is a complete and finite measure on the $\sigma$-field $\tilde{\Sigma}$. Moreover, from (2.12) we obtain immediately (2.15), (2.16).

This completes the proof.

Now, for each $x \in \tilde{\Omega}$, we construct two probability measures $P_{x}^{(\mathfrak{)})}$ $(i=1,2)$ on the $\sigma$-field $\tilde{\Sigma}$ by the following lemma:

LEмMа (2.4). Under the assumptions of Lemma (2.2), suppose that $P_{x}^{(i)}(\cdot)(x \in \tilde{\Omega} ; i=1,2)$ are the set-functions defined on $\tilde{\Sigma}$ by the formula

$$
P_{x}^{(i)}(\tilde{A})= \begin{cases}\int F_{i}(x, y) \tilde{\mu}(d y) & \text { for } x \in \Omega_{A}^{*} \backslash \Omega_{0}, \\ \chi_{\tilde{A}}(x) & \text { for } x \in A^{*} \cup \Omega_{0} \cup \Omega^{*},\end{cases}
$$

where $\tilde{A} \in \tilde{\Sigma}$, and $F_{i}(x, y)(i=1,2)$ are functions defined on $\left(\Omega_{A}^{*} \backslash \Omega_{0}\right) \times \tilde{\Omega}$ by the formula

$$
F_{i}(x, y)= \begin{cases}p(x, y) K(x, y) \quad \text { if }(x, y) \epsilon\left(\Omega_{A}^{*} \backslash \Omega_{0}\right) \times\left(\Omega \backslash \Omega_{0}\right), \\ p(x, y) K(x, y)+\frac{g_{i}(x) p(x, y)}{\mu\left(\Omega_{0}\right) g_{i}(y)} & \text { if }(x, y) \epsilon\left(\Omega_{A}^{*} \backslash \Omega_{0}\right) \times \Omega_{0}, \\ h_{i}(x) & \text { if }(x, y) \epsilon\left(\Omega_{A}^{*} \backslash \Omega_{0}\right) \times \Omega^{*} .\end{cases}
$$

Then

$1^{\circ}$ For a fixed $x \epsilon \tilde{\Omega}, i=1,2, P_{x}^{(i)}$ is a probability measure on $\dot{\Sigma}$.

$2^{\circ}$ For a fixed $x \epsilon \Omega_{A}^{*} \backslash \Omega_{0}, i=1,2, P_{x}^{(i)}$ is an absolutely continuous measure with respect to the measure $\tilde{\mu}\left(P_{x}^{(i)} \ll \tilde{\mu}\right)$. 
$3^{\circ}$ For a fixed $\tilde{A} \epsilon \tilde{\Sigma}$, the functions of $x: P_{x}^{(i)}(\tilde{A})(i=1,2)$ are $\tilde{\Sigma}-$ measurable on $\tilde{\Omega}$.

Proof. In the case $x \in A^{*} \cup \Omega_{0} \cup \Omega^{*}$, since for each $\tilde{A} \in \tilde{\Sigma}$ we have $P_{x}^{(i)}(\tilde{A})=\chi \tilde{A}(x)$ (see (2.19)), it is clear that $P_{x}^{(i)}$ is a probability measure on $\tilde{\Sigma}(i=1,2)$. Now we consider the case $x \in \Omega_{A}^{*} \backslash \Omega_{0}$.

From (2.7), (2.8), (2.20) and condition $\left(\mathrm{P}_{2}^{*}\right)$ we deduce

$$
F_{\imath}(x, y) \geqslant 0 \quad \text { for } y \in \tilde{\Omega}(\bmod \tilde{\mu}), x \in \Omega_{A}^{*} \backslash \Omega_{0} .
$$

Therefore, using the formula (see (2.19))

$$
P_{x}^{(i)}(A)=\int_{\tilde{\boldsymbol{A}}} F_{i}(x, y) \tilde{\mu}(d y) \quad\left(x \in \Omega_{A}^{*} \backslash \Omega_{0} ; \tilde{A} \in \tilde{\Sigma}\right)
$$

we conclude that $P_{x}^{(i)}\left(x \in \Omega_{A}^{*} \backslash \Omega_{0} ; i=1,2\right)$ are complete measures on $\tilde{\Sigma}$. Moreover, from $(2.22),(2.20),(2.15),(2.16),(2.5)$ it is not difficult to deduce

$$
P_{x}^{(j)}(\tilde{\Omega})=\int_{\tilde{\Omega}} F_{i}(x, y) \tilde{\mu}(d y)=1 \quad\left(x \in \Omega_{A}^{*} \backslash \Omega_{0} ; i=1,2\right)
$$

i. e. also in the case $x \in \Omega_{A}^{*} \backslash \Omega_{0}, P_{x}^{(i)}$ is a probability measure on $\tilde{\Sigma}$. The first assertion of the lemma is proved.

The second assertion is deduced directly from (2.22). For the proof of the third assertion, we first consider the case: $x \in A^{*} \cup \Omega_{0} \cup \Omega^{*}$. Then, if we fix $\tilde{A} \epsilon \tilde{\Sigma}, i=1,2$, then $P_{x}^{(i)}(\tilde{A})=\chi_{\tilde{A}}(x)$ (see (2.19)).

Therefore the functions $P_{x}^{(i)}(\tilde{A})$ of the variable $x$, are $\tilde{\Sigma}$-measurable on $A^{*} \cup \Omega_{0} \cup \Omega^{*}$.

In the case : $x \in \Omega_{A}^{*} \backslash \Omega_{0}$, we deduce from $(2.20)$ that $F_{i}(x, y)(i=1,2)$ are functions $\tilde{\Sigma} \times \tilde{\Sigma}$-measurable on $\left(\Omega_{A}^{*} \backslash \Omega_{0}\right) \times \tilde{\Omega}$. Therefore the functions $P_{x}^{(i)}(\tilde{A})$ (defined by formula $(2.22)$ ) are $\tilde{\Sigma}$-measurable also on $\Omega_{A}^{*} \backslash \Omega_{0}$. The lemma is proved.

Using Lemma (2.4) we construct two homogeneous Markov processes in the broad sense in the phase space $\tilde{\Omega}$ as follows:

Denote by $P_{i}(k, x, \tilde{A})(i=1,2 ; x \in \tilde{\Omega} ; \tilde{A} \epsilon \tilde{\Sigma})$ the families of functions defined by the formula:

$$
P_{i}(k, x, \tilde{A})=\int_{\tilde{\Omega}} P_{i}(k-1, y, \tilde{A}) P_{i}(1, x, d y) \quad(k=2,3, \ldots)
$$

where

$$
P_{\mathfrak{i}}(1, x, \tilde{A})=P_{x}^{(i)}(\tilde{A}) .
$$

From Lemma (2.3), by induction, we can easily prove the following conclusion:

(2.25) If we fix $k, x$, then $P_{i}(k, x, \tilde{A})(i=1,2)$ are probability measures on $\tilde{\Sigma}$. 
(2.26) If we fix $k, \tilde{A}$, then the functions of $x: P_{i}(k, x, \tilde{A})(i=1,2)$ are $\tilde{\Sigma}$-measurable on $\tilde{\Omega}$.

(2.27) For any $x, \bar{A}$, the families of functions $P_{i}(k, x, \tilde{A})(i=1,2)$ satisfy the Chapman-Kolmogorof equation

$$
P_{i}(k, x, \tilde{A})=\int_{\tilde{\Omega}} P_{i}\left(k_{1}, y, \tilde{A}\right) P_{i}\left(k_{2}, x, d y\right),
$$

where $k_{1}, k_{2}$ are natural numbers and $k_{1}+k_{2}=k$.

From conditions (2.25)-(2.27) we conclude that (see [5], p. 280) each family of functions $P_{i}(k, x, \tilde{A})(i=1,2)$ will be the transition probabilities of a homogeneous Markov process in the broad sense in the phase space $\tilde{\Omega}$.

DEFINITION (2.1). Each Markov process corresponding to the transition probabilities $(2.23$ ) (where $i=1,2$ ) will be called the $i$-th process.

For each $i$-th process, we consider its trajectories:

$$
x \rightarrow x_{1} \rightarrow x_{2} \rightarrow \ldots \rightarrow x_{l-1} \rightarrow x_{l} \rightarrow \ldots
$$

with the same initial state $x \in \Omega_{A}^{*} \backslash \Omega_{0}-$ where $P_{i}\left(1, x_{l}, \tilde{A}\right) \equiv P_{x_{l}}^{(i)}(\tilde{A})$ is the probability of transition from the state $x_{l}$ to a state $x_{t+1} \in \tilde{A}$.

Suppose that $\tilde{\Omega}_{x}^{(i)}$ is the space of trajectories (2.28) of the $i$-th process, $\tilde{\Sigma}_{x}^{(i)}=\tilde{\Sigma} \times \tilde{\Sigma} \times \ldots \times \tilde{\Sigma} \times \ldots$

Then the probability measure $\tilde{\mu}_{x}^{(i)}$ on the $\sigma$-field $\tilde{\Sigma}_{x}^{(i)}$ is completely defined by the transition probabilities $P_{i}(k, x, \cdot)$ according to the following formula (see [1], p. 174-175):

$$
\begin{aligned}
& \tilde{\mu}_{x}^{(i)}\left\{\tilde{A}_{1} \times \tilde{A}_{2} \times \ldots \times \tilde{A}_{l}\right\} \\
&= \int_{\tilde{A}_{1}} P_{i}\left(1, x, d x_{1}\right) \int_{\tilde{A}_{2}} P_{i}\left(1, x_{1}, d x_{2}\right) \ldots \int_{\tilde{A}_{l}} P_{i}\left(1, x_{l-1}, d x_{l}\right) \\
&\left(\tilde{A}_{i} \in \tilde{\Sigma} ; i=1,2, \ldots, l\right) .
\end{aligned}
$$

Write

$$
\Omega_{x}^{(i)}=\bigcup_{n=1}^{\infty} \Omega_{x}^{(i)}[n] \quad\left(x \in \Omega_{A}^{*} \backslash \Omega_{0}\right),
$$

where $\Omega_{x}^{(i)}[n]$ is the subspace of $\Omega_{x}^{(i)}$ consisting of trajectories

$$
x \rightarrow x_{1} \rightarrow \ldots \rightarrow x_{n} \quad\left(x_{n} \in A^{*} \cup \Omega_{0} \cup \Omega^{*} ; x, x_{1}, \ldots, x_{n-1} \epsilon \Omega_{A}^{*} \backslash \Omega_{0}\right)
$$

with fixed length $n$ of the $i$-th process.

Then $\Omega_{x}^{(i)}$ is the subspace of $\tilde{\Omega}_{x}^{(i)}$ consisting of trajectories

$$
x \rightarrow x_{1} \rightarrow \ldots \rightarrow x_{l} \quad\left(x_{l} \in A^{*} \cup \Omega_{0} \cup \Omega^{*} ; x, x_{1}, \ldots, x_{l-1} \in \Omega_{A}^{*} \backslash \Omega_{0}\right)
$$

with finite length of the $i$-th process. 
We have the following lemma:

LEMMA (2.5). Under the assumptions of Lemma (2.2) and condition $\left(\mathrm{P}_{3}\right)$, suppose that $\xi^{(i)}(x)=f^{(i)}\left(x ; x_{1}, \ldots, x_{l}\right)$ is the function defined on $\Omega_{x}^{(i)}$ by the formula

$$
\xi^{(i)}(x)=f^{(i)}\left(x ; x_{1}, \ldots, x_{l}\right) \equiv\left\{\begin{array}{lr}
\frac{g_{i}\left(x_{l}\right)}{p\left(x, x_{1}\right) \ldots p\left(x_{l-1} x_{l}\right)} & \text { if } x_{l} \in \Omega_{0}, \\
0 & \text { if } x_{l} \in \Omega_{\mathbf{1}} \\
\left(x \in \Omega_{A}^{*} \backslash \Omega_{0} ;\right. & i=1,2),
\end{array}\right.
$$

where $\Omega_{1}=A^{*} \cup \Omega_{0} \cup \Omega^{*}$.

Then we have

$$
\tilde{\mu}_{x}^{(i)}\left\{\Omega_{x}^{(i)}\right\}=1 \quad\left(x \in \Omega_{\Delta}^{*} \backslash \Omega_{0} ; i=1,2\right) .
$$

Moreover, $\xi^{(i)}(x)$ is a random variable defined on the probability space $\left(\tilde{\Omega}_{x}^{(i)}, \tilde{\Sigma}_{x}^{(i)}, \tilde{\mu}_{x}^{(i)}\right)$.

Proof. Write

$$
q^{(i)}=\inf _{x \in \Omega_{A}^{*} \backslash \Omega_{0}}\left\{P_{i}\left(1, x, \Omega_{0} \cup \Omega_{1}\right)\right\}
$$

From $(2.19),(2.20),(2.5),(2.15)$, it is easy to deduce

$$
\begin{aligned}
P_{i}\left(1, x, \Omega_{0} \cup \Omega_{1}\right) & \equiv P_{x}^{(i)}\left\{\Omega_{0} \cup \Omega_{1}\right\} \\
& =1-\int_{\Omega \backslash \Omega_{0}} p(x, y) K(x, y) \mu(d y) \quad\left(x \in \Omega_{\Delta}^{*} \backslash \Omega_{0}\right) .
\end{aligned}
$$

Therefore, from (2.35) and condition $\left(\mathrm{P}_{1}^{*}\right)$ we have

$$
1 \geqslant q^{(i)}=1-\sup _{s \in \Omega_{A}^{*} \backslash \Omega_{0}}\left\{\int_{\Omega} p(x, y) K(x, y) \mu(d y)\right\}>0 .
$$

Note that (see $(2.19),(2.23)$ )

$$
P_{i}(k, y, \tilde{A})=\chi \tilde{A}(y) \quad\left(y \in \Omega_{0} \cup \Omega_{1} ; \tilde{A} \in \tilde{\Sigma} ; k=1,2, \ldots\right) .
$$

Therefore, from (2.23) we get

$$
\begin{array}{r}
P_{i}\left(k, x, \Omega_{A}^{*} \backslash \Omega_{0}\right)=\int_{\Omega_{A}^{*} \backslash \Omega_{0}} P_{i}\left(k-1, y, \Omega_{A}^{*} \backslash \Omega_{0}\right) P_{i}(1, x, d y) \\
\left(x \in \Omega_{A}^{*} \backslash \Omega_{0} ; k=1,2,3, \ldots\right) .
\end{array}
$$

Hence, by induction,

$$
P_{\boldsymbol{i}}\left(k, x, \Omega_{\Delta}^{*} \backslash \Omega_{0}\right) \leqslant\left(1-q^{(i)}\right)^{k} \quad\left(x \epsilon \Omega_{\Delta}^{*} \backslash \Omega_{0} ; k=1,2, \ldots\right) .
$$

Therefore, from (2.37) we conclude

$$
\begin{aligned}
& \lim _{k \rightarrow \infty} P_{i}\left(k, x, \Omega_{0} \cup \Omega_{1}\right)=1-\lim _{k \rightarrow \infty} P_{i}\left(k, x, \Omega_{\Delta}^{*} \backslash \Omega_{0}\right)=1 \\
&\left(x \in \Omega_{\Delta}^{*} \backslash \Omega_{0} ; i=1,2\right) .
\end{aligned}
$$


From (2.29) we obtain

$$
\begin{array}{r}
\tilde{\mu}_{x}^{(i)}\left\{\Omega_{x}^{(i)}[n]\right\}=\int_{\Omega_{A}^{*} \backslash \Omega_{0}} \tilde{\mu}_{x_{1}}^{(i)}\left\{\Omega_{x_{1}}^{(i)}[n-1]\right\} \\
P_{i}\left(1, x, d x_{1}\right) \\
\\
\quad\left(x \in \Omega_{A}^{*} \backslash \Omega_{0} ; n \geqslant 2\right) .
\end{array}
$$

Applying again the induction, from $(2.38),(2.23)$ we have

$$
\begin{array}{r}
\tilde{\mu}_{x}^{(i)}\left\{\Omega_{x}^{(i)}[n]\right\}=P_{i}\left(n, x, \Omega_{0} \cup \Omega_{1}\right)-P_{i}\left(n-1, x, \Omega_{0} \cup \Omega_{1}\right) \\
\left(x \in \Omega_{A}^{*} \backslash \Omega_{0} ; n \geqslant 2\right)
\end{array}
$$

or

$$
\sum_{n=1}^{k} \tilde{\mu}_{x}^{(i)}\left\{\Omega_{x}^{(i)}[n]\right\}=P_{i}\left(k, x, \Omega_{0} \cup \Omega_{1}\right) \quad\left(x \in \Omega_{A}^{*} \backslash \Omega_{0}\right) .
$$

Then from (2.30), (2.41) we deduce

$$
\tilde{\mu}_{x}^{(i)}\left\{\Omega_{x}^{(i)}\right\}=\sum_{n=1}^{\infty} \tilde{\mu}_{x}^{(i)}\left\{\Omega_{x}^{(i)}[n]\right\}=\lim _{k \rightarrow \infty} \sum_{n=1}^{k} \tilde{\mu}_{x}^{(i)}\left\{\Omega_{x}^{(i)}[n]\right\}=1
$$

i. e. we obtain (2.34).

It is evident that the functions $g_{i}(x)(i=1,2)$ are $\tilde{\Sigma}$-measurable and bounded on $\Omega(\bmod \mu$ ) (see Lemma $(2.2)$ ) and $p(x, y)$ is $\tilde{\Sigma} \times \tilde{\Sigma}$-measurable and bounded on $\Omega \times \Omega$. Therefore from condition $\left(\mathbf{P}_{3}\right)$, we conclude that the function $f^{(i)}\left(x ; x_{1}, \ldots, x_{l}\right)$ defined by formula $(2.33)$ is $\tilde{\Sigma}_{x}^{(i)}$-measurable and finite on $\Omega_{x}^{(i)}\left(\bmod \tilde{\mu}_{x}^{(i)}\right)$. Notice that $\tilde{\mu}_{x}^{(i)}\left\{\tilde{\Omega}_{x}^{(i)}\right\}=1$, therefore from (2.34) we deduce that the function $f^{(i)}\left(x ; x_{1}, \ldots, x_{l}\right)$ is also $\tilde{\Sigma}_{x}^{(i)}$ measurable and finite on $\tilde{\Omega}_{x}^{(i)}\left(\bmod \tilde{\mu}_{x}^{(i)}\right)$.

I. e. $\xi^{(i)}(x)=f^{(i)}\left(x ; x_{1}, \ldots, x_{l}\right)\left(x \in \Omega_{A}^{*} \backslash \Omega_{0}\right)$ is a random variable defined on the probability space $\left(\tilde{\Omega}_{x}^{(i)}, \tilde{\Sigma}_{x}^{(i)}, \tilde{\mu}_{x}^{(i)}\right)$.

This completes the proof.

Concerning the solution of equation (1.1) by the Monte-Carlo method, we prove the following theorem:

THEOREM (2.1). Under assumptions (A), (B), (C), $\left(\mathbf{P}_{1}\right)-\left(\mathbf{P}_{3}\right)$, suppose that $\xi^{(i)}(x)\left(i=1,2 ; x \in \Omega_{A}^{*} \backslash \Omega_{0}\right)$ are random variables defined in Lemma (2.5). Then the expected values $M \xi^{(i)}(x)$ exist and are finite, and we have

$$
M \xi^{(i)}(x)=u^{(i)}(x) \quad\left(x \in \Omega_{A}^{*} \backslash \Omega_{0} ; i=1,2\right),
$$

where $u^{(i)}(x)$ is the solution of the equation

$$
u^{(i)}(x)-\int_{\Omega} K(x, y) u^{(i)}(y) \mu(d y)=g_{i}(x) \quad(x \in \Omega)
$$


Moreover,

$$
M \xi^{(1)}(x)+M \xi^{(2)}(x)=u(x) \quad\left(x \in \Omega \backslash \Omega_{0}(\bmod \mu)\right)\left({ }^{5}\right),
$$

where $u(x)$ is the solution in $L_{\infty}(\Omega)$ of equation (1.1).

Proof. By condition (C), equation (2.47) is equivalent to the following equations :

$$
\begin{gathered}
u^{(i)}(x)=g_{i}(x) \quad\left(x \in \Omega_{0}(\bmod \mu)\right), \\
u^{(i)}(x)-\int_{\Omega \backslash \Omega_{0}} K(x, y) u^{(i)}(y) \mu(d y)=G_{i}(x) \quad\left(x \in \Omega \backslash \Omega_{0}\right),
\end{gathered}
$$

where

$$
G_{i}(x)=\int_{\Omega_{0}} K(x, y) g_{i}(y) \mu(d y)+g_{i}(x) \quad\left(x \in \Omega \backslash \Omega_{0}\right)
$$

Let

$$
u_{n}^{(i)}(x)=\left[T^{n-1} G_{i}\right](x) \quad\left(x \in \Omega_{A}^{*} \backslash \Omega_{0} ; n=1,2, \ldots\right),
$$

where $T$ is the integral operator defined by the formula

$$
[T f](x)=\int_{\Omega \backslash \Omega_{0}} K(x, y) f(y) \mu(d y)
$$

Since $T \epsilon\left[L_{\infty}\left(\Omega \backslash \Omega_{0}\right) \rightarrow L_{\infty}\left(\Omega \backslash \Omega_{0}\right)\right]$ and $G_{i} \epsilon L_{\infty}\left(\Omega \backslash \Omega_{0}\right)$, therefore from (2.51) we conclude that $u_{n}^{(i)} \in L_{\infty}\left(\Omega \backslash \Omega_{0}\right)$.

In the case: $x \in \Omega_{A}^{*} \backslash \Omega_{0}$, we have

$$
\begin{gathered}
\frac{d P_{x}^{(i)}}{d \tilde{\mu}}(\cdot)=F_{i}(x, \cdot) \quad(\text { see }(2.19)), \\
\left.P_{i}(1, x, \cdot) \equiv P_{x}^{(i)}(\cdot) \ll \tilde{\mu} \quad \text { (see Lemma }(2.4)\right) .
\end{gathered}
$$

Then, from $(2.50),(2.20)$ we immediately deduce

$$
G_{i}(x)=\int_{\Omega_{0}} \frac{g_{i}(y)}{p(x, y)} P_{i}(1, x, d y) \quad\left(x \in \Omega_{A}^{*} \backslash \Omega_{0}\right) .
$$

Analogously, from (2.51) we obtain

$$
\begin{gathered}
(2.56) u_{n}^{(i)}(x)=\underbrace{\int \ldots \int_{(n-1)}}_{\Omega_{A}^{*} \backslash \Omega_{0} \Omega_{A^{*} \backslash \Omega_{0}}} \frac{G_{i}\left(x_{n-1}\right)}{p\left(x, x_{1}\right) \ldots p\left(n_{n-2}, x_{n-1}\right)} P_{i}\left(1, x_{n-2}, d x_{n-1}\right) \ldots \\
\ldots P_{i}\left(1, x, d x_{1}\right) \quad\left(x \in \Omega_{A}^{*} \backslash \Omega_{0}\right) .
\end{gathered}
$$

(5) From condition (C) we deduce that

$$
u(x)=g(x) \quad \text { for } x \in \Omega_{0}(\bmod \mu) .
$$


Therefore, from (2.55), (2.33), (2.29) we have

$$
u_{n}^{(i)}(x)=\int_{\Omega_{x}^{(i)}[n]} f^{(i)} d \tilde{\mu}_{x}^{(i)} \quad\left(x \in \Omega_{A}^{*} \backslash \Omega_{0} ; n=1,2, \ldots\right) .
$$

Let

$$
\vartheta_{n}^{(i)}(x)=\left[T_{+}^{n-1} G_{i}^{+}\right](x) \quad\left(x \in \Omega_{A}^{*} \backslash \Omega_{0} ; n=1,2, \ldots\right)
$$

where

$G_{i}^{+}(x)=\int_{\Omega_{0}} K(x, y)\left|g_{i}(y)\right| \mu(d y)+\frac{g_{i}(x)}{\mu\left(\Omega_{0}\right)} \int_{\Omega_{0}} \operatorname{sgn}\left\{g_{i}(y)\right\} \mu(d y) \quad\left(x \in \Omega_{A}^{*} \backslash \Omega_{0}\right)$.

Since $T_{+} \in\left[L_{\infty}\left(\Omega \backslash \Omega_{0}\right) \rightarrow L_{\infty}\left(\Omega \backslash \Omega_{0}\right)\right]$ (by condition (A)) and $G_{i}^{+}$ $\epsilon L_{\infty}\left(\Omega \backslash \Omega_{0}\right)$, therefore from (2.58) we deduce that $\vartheta_{n}^{(i)} \epsilon L_{\infty}\left(\Omega \backslash \Omega_{0}\right)$. In analogy with $(2.55)$, from $(2.59)$ and condition $\left(B^{*}\right)$ we have

$$
G_{i}^{+}(x)=\int_{\Omega_{0}}\left|\frac{g_{i}(y)}{p(x, y)}\right| P_{i}(1, x, d y) \quad\left(x \in \Omega_{A}^{*} \backslash \Omega_{0}\right) .
$$

From (2.58) and condition $\left(\mathrm{P}_{2}^{*}\right)$, it is easy to see that:

$$
\begin{array}{r}
(2.61) \quad \ldots \int_{\Omega_{A}^{-} \backslash \Omega_{0} \underbrace{*}_{(n-1)}}^{\vartheta_{n}^{(i)}(x)} \int_{\Omega^{-} \backslash \Omega_{0}} \frac{G_{i}^{+}\left(x_{n-1}\right)}{\left|p\left(x, x_{1}\right) \ldots p\left(x_{n-2}, x_{n-1}\right)\right|} P_{i}\left(1, x_{n-2}, d x_{n-1}\right) \ldots \\
\ldots P_{i}\left(1, x, d x_{1}\right) \quad\left(x \in \Omega \backslash \Omega_{0}\right) .
\end{array}
$$

Then, by (2.60), (2.33), (2.29) we get

$$
\vartheta_{n}^{(i)}(x)=\int_{\Omega_{x}^{(i)}[n]}\left|f^{(i)}\right| d \tilde{\mu}_{x}^{(i)} \quad\left(x \in \Omega_{A}^{*} \backslash \Omega_{0} ; n=1,2, \ldots\right) .
$$

Consequently, by condition (A), that the series

$$
\sum_{n=1}^{\infty} \int_{\Omega_{x}^{(i)}[n]}\left|f^{(i)}\right| d \tilde{\mu}_{x}^{(i)}=\sum_{n=1}^{\infty} \vartheta_{n}^{(i)}(x) \equiv \sum_{n=0}^{\infty}\left[T_{+}^{n} G_{i}^{+}\right](x)
$$

converges in $L_{\infty}\left(\Omega \backslash \Omega_{0}\right)$ and thus, by (2.57), also the series

$$
\sum_{n=1}^{\infty} \cdot \int_{\Omega_{x}^{(i)}[n]}\left|f^{(i)}\right| d \tilde{\mu}_{x}^{(i)}=\sum_{n=1}^{\infty} \vartheta_{n}^{(i)}(x) \equiv \sum_{n=0}^{\infty}\left[T_{+}^{n} G_{i}^{+}\right](x)
$$

converges in $L_{\infty}\left(\Omega \backslash \Omega_{0}\right)$. 
Note that (2.64) is the Neumann series for equation $\left(2.49^{\prime}\right)$. Therefore, from the convergence of this series in the $B$-space $L_{\infty}\left(\Omega \backslash \Omega_{0}\right)$ it follows that the solution $u^{(i)}(x)$ of equation $\left(2.49^{\prime}\right)$ exists and is unique in $L_{\infty}\left(\Omega \backslash \Omega_{0}\right)$.

Moreover, we have

$$
u^{(j)}(x)=\sum_{n=1}^{\infty} \int_{\Omega_{x}^{(i)}[n]} f^{(i)} d \tilde{\mu}_{x}^{(i)}=\sum_{n=1}^{\infty} u_{n}^{(i)}(x) \quad\left(x \in \Omega_{A}^{*} \backslash \Omega_{0}\right) .
$$

Then, applying Lebesgue's theorem, from the convergence of the series (2.63) we obtain

$$
\int_{\Omega_{x}^{(i)}} f^{(i)} d \tilde{\mu}_{x}^{(i)}=u^{(i)}(x) \quad\left(x \in \Omega_{A}^{*} \backslash \Omega_{0}\right)
$$

It is known that $\tilde{\mu}_{x}^{(i)}\left\{\tilde{\Omega}_{x}^{(i)} \backslash \Omega_{x}^{(i)}\right\}=0$ (by $\left.(2.34)\right)$. Therefore, from (2.66) we have

$$
M \xi^{(i)}(x) \equiv \int_{\tilde{\Omega}_{x}^{(i)}} f^{(i)} d \tilde{\mu}_{x}^{(i)}=\int_{\Omega_{x}^{(i)}} f^{(i)} d \tilde{\mu}_{x}^{(i)}=u^{(i)}(x) \quad\left(x \in \Omega_{A}^{*} \backslash \Omega_{0}\right)
$$

i. e. (2.46). Then, from (2.4) we obtain (2.48). This completes the proof.

3. The probability model for the evaluation of the functional (1.2). Suppose that $K(x)$ is a function satisfying the following conditions

$$
\begin{gathered}
K(x) \geqslant 0 \quad \text { if } x \in \tilde{\Omega}(\bmod \tilde{\mu}) ; \\
0<K(x)<+\infty \quad \text { if } x \in \Omega(\bmod \mu)\left({ }^{6}\right) ; \\
\int_{\tilde{\Omega}} K(x) \tilde{\mu}(d x)=1 .
\end{gathered}
$$

Let $P(\cdot)$ be a probability measure defined on the $\sigma$-field $\tilde{\Sigma}$ by the formula

$$
P(\tilde{A})=\int_{\tilde{\boldsymbol{A}}} K(y) \tilde{\mu}(d y) \quad(\tilde{A} \epsilon \tilde{\Sigma}) .
$$

For each $i$-th process $(i=1,2)$, we consider the trajectories

$$
x_{0} \rightarrow x_{1} \rightarrow x_{2} \rightarrow \ldots \rightarrow x_{l-1} \rightarrow x_{l} \rightarrow \ldots
$$

with the initial probability distribution $P(\cdot)$

Suppose that $\tilde{\Omega}^{(i)}$ is the sample space of the trajectories (3.2) of the $i$-th process and $\tilde{\Sigma}^{(i)}=\tilde{\Sigma} \times \tilde{\Sigma}_{x}^{(i)}$. Then the probability measure $\tilde{\mu}^{(i)}$

(6) Assumption $\left(K_{2}\right)$ can be replaced by the following one:

$$
0<K(x)<+\infty \quad \text { if } x \in \Omega_{\varphi}(\bmod \mu) \text {, }
$$

where $\Omega_{\varphi}=\{x: \varphi(x) \neq 0\} \subset \Omega$. 
on $\left(\tilde{\Omega}^{(i)}, \tilde{\Sigma}^{(i)}\right)$ is completely defined (by the initial probability distribution $P(\cdot)$ and the transition probabilities $\left.P_{i}(k, x, \cdot)\right)$ by the formula (see [1], p. 174)

$$
\begin{aligned}
\tilde{\mu}^{(i)}\left\{\tilde{A}_{0}\right. & \left.\times \tilde{A}_{1} \times \ldots \times \tilde{A_{l}}\right\} \\
= & \int_{\tilde{A_{0}}} P\left(d x_{0}\right) \int_{\tilde{A_{1}}} P_{i}\left(1, x_{0}, d x_{1}\right) \ldots \int_{\tilde{A}_{l}} P_{i}\left(1, x_{l-1}, d x_{l}\right) \\
& \left(\tilde{A}_{k} \in \tilde{\Sigma} ; k=0,1, \ldots, l\right) .
\end{aligned}
$$

Suppose

$$
\Omega^{(i)}=\bigcup_{n=0}^{\infty} \Omega^{(i)}[n]
$$

where $\Omega^{(i)}[n]$ is the subspace of $\tilde{\Omega}^{(i)}$ consisting of trajectories

$$
x_{0} \rightarrow x_{1} \rightarrow \ldots \rightarrow x_{n} \quad\left(x_{n} \in \Omega_{0} \cup \Omega_{1} ; x_{0}, x_{1}, \ldots, x_{n-1} \in \Omega_{A}^{*} \backslash \Omega_{0}\right)
$$

with fixed length $n$ of the $i$-th process.

Then $\Omega^{(i)}$ is the subspace of $\tilde{\Omega}^{(i)}$ consisting of trajectories

$$
\dot{x}_{0} \rightarrow x_{1} \rightarrow \ldots \rightarrow x_{l} \quad\left(x_{l} \in \Omega_{0} \cup \Omega_{1} ; x_{0}, x_{1}, \ldots x_{l-1} \in \Omega_{A}^{*} \backslash \Omega_{0}\right)
$$

with finite length of the $i$-th process.

From (3.3), (2.29) we deduce

$$
\begin{gathered}
\tilde{\mu}^{(i)}\left\{\Omega^{(i)}[0]\right\}=\int_{\Omega_{1} \cup \Omega_{0}} P\left(d x_{0}\right), \\
\tilde{\mu}^{(i)}\left\{\Omega^{(i)}[n]\right\}=\int_{\Omega_{A}^{*} \backslash \Omega_{0}} \tilde{\mu}_{x_{0}}^{(i)}\left\{\Omega_{x_{0}}^{(i)}[n]\right\} P\left(d x_{0}\right) \quad(n=1,2, \ldots) .
\end{gathered}
$$

Therefore, by Lebesgue's theorem and (2.34) we have

$$
\sum_{n=1}^{\infty} \tilde{\mu}^{(i)}\left\{\Omega^{(i)}[n]\right\}=\int_{\Omega_{A}^{*} \backslash \Omega_{0}} \tilde{\mu}_{x_{0}}^{(i)}\left\{\Omega_{x_{0}}^{(i)}\right\} P\left(d x_{0}\right)=\int_{\Omega_{A}^{*} \backslash \Omega_{0}} P\left(d x_{0}\right)
$$

From (3.7), (3.9) and condition $\left(\mathbf{K}_{3}\right)$ we obtain

$$
\tilde{\mu}^{(i)}\left\{\Omega^{(i)}\right\}=\sum_{n=0}^{\infty} \tilde{\mu}^{(i)}\left\{\Omega^{(i)}[n]\right\}=\int_{\bar{\Omega}} P\left(d x_{0}\right)=1 .
$$

It is known that $\tilde{\mu}^{(i)}\left\{\tilde{\Omega}^{(i)}\right\}=1$. Then, from (3.10) we have

$$
\tilde{\mu}^{(i)}\left\{\tilde{\Omega}^{(i)} \backslash \Omega^{(i)}\right\}=0 \quad(i=1,2) .
$$

Suppose that $\eta^{(i)}=F^{(i)}\left(x_{0}, x_{1}, \ldots, x_{l}\right)$ is a function defined on $\Omega^{(i)}$ by the formula

$$
\eta^{(i)}=F^{(i)}\left(x_{0}, x_{1}, \ldots, x_{l}\right) \equiv \begin{cases}\frac{\psi\left(x_{0}\right) g_{i}\left(x_{l}\right)}{p\left(x_{0}, x_{1}\right) \ldots p\left(x_{l-1}, x_{l}\right)} & \text { for } x_{l} \epsilon \Omega_{0} ; l \geqslant 1, \\ \psi\left(x_{0}\right) g_{i}\left(x_{0}\right) & \text { for } x_{0} \epsilon \Omega_{0}, \\ 0 & \text { for } x_{l} \epsilon \Omega_{1},\end{cases}
$$


where

$$
\psi(x)=\frac{\varphi(x)}{K(x)} \quad(x \in \Omega)
$$

We know that $\varphi, K \in L_{1}(\Omega)$ and $K(x) \neq 0$ on $\Omega$ (by condition $\left(\mathbf{K}_{2}\right)$ ) i. e. $\psi(x)$ is a $\tilde{\Sigma}$-measurable and finite function on $\Omega(\bmod \mu)$. Therefore from Lemma $(2.5)$ we conclude that $\eta^{(i)}=F^{(i)}\left(x_{0}, x_{1}, \ldots, x_{l}\right)$ is a $\tilde{\Sigma}^{(i)}$ measurable and finite function on $\Omega^{(i)}\left(\bmod \tilde{\mu}^{(i)}\right)$. Thus it follows from (3.11) that this function is also $\tilde{\Sigma}^{(i)}$-measurable and finite on $\tilde{\Omega}^{(i)}\left(\bmod \tilde{\mu}^{(i)}\right)$, i. e. $\eta^{(i)}=F^{(i)}\left(x_{0}, x_{1}, \ldots, x_{l}\right)$ is a random variable defined on the probability space $\left(\tilde{\Omega}^{(i)}, \tilde{\Sigma}^{(i)}, \tilde{\mu}^{(i)}\right)$.

For the estimation of the value of functional (1.2) by the Monte-Carlo method, we prove the following theorem:

THEOREM (3.1). Under assumptions (A), (B), (C), $\left(\mathbf{P}_{\mathbf{1}}\right)-\left(\mathbf{P}_{\mathbf{3}}\right),\left(\mathbf{K}_{\mathbf{1}}\right)-\left(\mathbf{K}_{\mathbf{3}}\right)$ and $\varphi \epsilon L_{1}(\Omega)$, suppose that $\eta^{(i)}(i=1,2)$ are random variables defined by formula (3.12). Then the expected values $M \eta^{(i)}(i=1,2)$ exist and are finite.

We have

$$
M \eta^{(i)}=\left(u^{(i)}, \varphi\right) \equiv \int_{\Omega} u^{(i)}(x) \varphi(x) \mu(d x) \quad(i=1,2)
$$

where $u^{(i)}(x)$ is the solution of equation (2.47).

Moreover,

$$
M \eta^{(1)}+M \eta^{(2)}=(u, \varphi) \equiv \int_{\Omega} u(x) \varphi(x) \mu(d x)
$$

where $u(x)$ is the solution of equation (1.1).

Proof. Denote

$$
U_{n}^{(i)}=\left(u_{n}^{(i)}, \varphi\right)_{\Omega_{A}^{*} \backslash \Omega_{0}} \equiv \int_{\Omega_{A}^{*} \backslash \Omega_{0}} u_{n}^{(i)}(x) \varphi(x) \mu(d x) \quad(n=1,2, \ldots)\left({ }^{7}\right),
$$

where $u_{n}^{(i)}(x)$ is defined by formula (2.51).

Since $u_{n}^{(i)} \in L_{\infty}\left(\Omega \backslash \Omega_{0}\right), \varphi \in L_{1}(\Omega)$, then $U_{n}^{(1)}$ is finite.

We know that $\frac{d P}{d \tilde{\mu}}(\cdot)=K(\cdot)$ (see (3.1)). Therefore from (2.57), (3.13), (3.16) we have

$$
U_{n}^{(i)}=\int_{\Omega_{\Lambda}^{*} \backslash \Omega_{0}} \int_{\Omega_{x}^{(i)}[n]} \psi(x) f^{(i)}\left(x ; x_{1}, \ldots, x_{n}\right) d \tilde{\mu}_{x}^{(i)} P(d x) .
$$

(7) The symbol $\left(f_{1}, f_{2}\right)_{A}$ denotes the value of the integral $\int_{\boldsymbol{A}} f_{1}(x) f_{2}(x) \mu(d x)$. 
Hence and from (2.29), (3.3), (3.12) we deduce

$$
U_{n}^{(i)}=\int_{\Omega^{(i)}[n]} F^{(i)} d \tilde{\mu}^{(i)} \quad(i=1,2 ; n=1,2, \ldots) .
$$

Analogously, denoting

$$
V_{n}^{(i)}=\left(\vartheta_{n}^{(i)},|\varphi|\right)_{\Omega_{A}^{*} \backslash \Omega_{0}} \equiv \int_{\Omega_{A}^{*} \backslash \Omega_{0}} \vartheta_{n}^{(i)}(x)|\varphi(x)| \mu(d x)
$$

we get

$$
V_{n}^{(i)}=\int_{\Omega^{(i)}[n]}\left|F^{(i)}\right| d \tilde{\mu}^{(i)} \quad(i=1,2 ; n=1,2, \ldots)
$$

Since $\vartheta_{n}^{(i)}(x) \geqslant 0$ on $\Omega_{A}^{*} \backslash \Omega_{0}$ (by (2.62)) and the series (2.63) converges in $L_{\infty}\left(\Omega \backslash \Omega_{0}\right)$, then applying Lebesgue's theorem, from (3.18), (3.19) we obtain

$$
\sum_{n=1}^{\infty} \int_{\Omega^{(i)}[n]}\left|F^{(i)}\right| d \tilde{\mu}^{(i)}=\int_{\Omega_{A}^{*} \backslash \Omega_{0}} \sum_{n=1}^{\infty} \vartheta_{n}^{(i)}(x)|\varphi(x)| \mu(d x),
$$

i. e. the series $\sum_{n=1}^{\infty} V_{n}^{(i)}=\sum_{n=1}^{\infty} \int_{\Omega(i)}\left|F^{(i)}\right| d \tilde{\mu}^{(i)}$ converges $\left(^{8}\right)$. Therefore, from (3.11) we conclude that the expected values $M \eta^{(i)}(i=1,2)$ exist and are finite.

Further, we have

$$
M \eta^{(i)} \equiv \int_{\tilde{\Omega}(i)} F^{(i)} d \tilde{\mu}^{(i)}=\int_{\Omega^{(i)}} F^{(i)} d \tilde{\mu}^{(i)}=\sum_{n=0}^{\infty} \int_{\Omega^{(i)}[n]} F^{(i)} d \tilde{\mu}^{(i)} .
$$

From (3.3), (3.12), (2.49) we deduce

$$
\int_{\Omega^{(i)}[0]} F^{(i)} d \tilde{\mu}^{(i)}=\int_{\Omega_{0}} \psi(x) g_{i}(x) P(d x)=\left(g_{i}, \varphi\right)_{\Omega_{0}}=\left(u^{(i)}, \varphi\right)_{\Omega_{0}} .
$$

Moreover, from (3.16), (3.17), (2.65) we have

$$
\sum_{n=1}^{\infty} \int_{\Omega(i)[n]} F^{(i)} d \tilde{\mu}^{(i)}=\int_{\Omega_{A}^{*} \backslash \Omega_{0}} \sum_{n=1}^{\infty} u_{n}^{(i)}(x) \varphi(x) \mu(d x)=\left(u^{(i)}, \varphi\right)_{\Omega \backslash \Omega_{0}} \cdot
$$

From (3.21)-(3.23) we deduce (3.14). Therefore, from (2.4), (2.47), it is easy to have (3.15).

This completes the proof.

The author wishes to express his gratitude to Professor B. Bojarski for many valuable discussions and remarks.

(8) Therefore, the series $\sum_{n=1}^{\infty} U_{n}^{(i)}=\sum_{n=1}^{\infty} \int_{\Omega^{(i)}[n]} F^{(i)} d \tilde{\mu}^{(i)}$ also converges. 


\section{References}

[1] J. L. Doob, Stochastic processes, Moscow 1956 (in Russion).

[2] Nguyen Quy Hy, Tap san Toan Ly, 9, No, 1-2, p. 45-49, Hanoi 1971 (in Vietnamese).

[3] - Thong bao Khoßhoc Dai hoc Tonghop Hanoi No. 4 (1971) p. 108-122 (in Vietnamese).

[4] J. Spanier, Naval Reactors Phys. Handbook, 1 (1964), USAES, p. 1441-1461.

[5] И. М. Гихман, А. В. Скороход, Вөедение в теорию случайных пройесов, Warsaw 1968 (in Polish).

[6] С. М. Ермаков, В. С. Нефедов, ДАН. СССР 202 (1972), No. 1, p. 27-29.

[7] В. Г. Золотухин, С. М. Ермаков, Вопр. Фив. Защиты реакторов, Вып. 2, p. 171-182, Москва 1963.

[8] Б. А. Каргин, Г. А. Михалов, Ж. Вычисл. Матем. и Матем. Фив. 12 No. 1 (1972), p. 150-158.

[9] Г. А. Миха лов. Ж. Вычисл. Матем. и Матем. Фив. 8, o. 3 (1968), p. 590-599.

[10] - Ж. Внчисл. Матем. и Матем. Фив. 8, о. 5 (1968), р. 1085-1093.

[11] А. И. Хисамутдинов, Ж. Вычисл. Матем. и Матем. Фив. 8, №. 2 (1968), p. 467-471.

[12] - Ж. Внчисл. Матем. и Матем. Фия. 10 No. 4 (1970), p. 999-1005.

[13] - Ж. Внчисл. Матем. и Матем. Фив. 10, о. 5 (1970), p. 1269-1280.

INSTITUTE OF MATHEMATICS, UNIVERSITY, WARSAW

Reģu par la Rédaction le 30. 1. 1973 